\title{
Prevalencia de ansiedad y depresión en pacientes con enfermedad cardiovascular durante la pandemia COVID- 19
}

\author{
Prevalence of anxiety and depression in patients with cardiovascular disease during the \\ COVID-I 9 pandemic
}

\section{Gustavo H. Cerezo',Augusto Vicario'}

\section{Resumen}

Introducción: La pandemia COVID-19 y las medidas sanitarias implementadas impactaron sobre la salud mental de las poblaciones más vulnerables. Objetivo: Conocer la prevalencia de ansiedad y depresión en pacientes con enfermedad cardiovascular y/o factores de riesgo después de finalizada la cuarentena y compararla con la prevalencia durante la cuarentena y antes de la pandemia COVID-19. Métodos: Se envió un cuestionario on-line a los 150 días de finalizada la cuarentena. Se utilizó la escala de Ansiedad-Depresión Hospitalaria (HAD, por sus siglas en inglés) y se registraron los datos clínicos y demográficos. Los resultados fueron comparados con las muestras obtenidas durante la cuarentena y el estudio EPICA realizado en 2016 antes de la pandemia COVID-19. Resultados: Las muestras incluyeron 1076, 3542 y 1035 pacientes respectivamente. La prevalencia de ansiedad fluctuó pero no cambió $(20.8 \%$, $13.5 \%$ y $21 \%$ respectivamente). La depresión incrementó su prevalencia $(9.8 \%, 16.7 \%$ y $19.7 \%$ respectivamente). La depresión se asoció con la enfermedad coronaria y las conductas de riesgo (fumar cigarrillos y sedentarismo). Conclusión: La depresión duplicó su prevalencia durante la pandemia COVID-I 9 en pacientes con enfermedad cardiovascular y/o factores de riesgo. Los efectos de la pandemia parecen extenderse más allá de la duración de la cuarentena.

Palabras claves: Ansiedad - Depresión - COVID-19 - HAD - Enfermedad cardiovascular.

\begin{abstract}
Introduction: The COVID-19 pandemic and the sanitary measures implemented had an impact on the mental health of the most vulnerable populations. Aims: To know the prevalence of anxiety and depression in patients with cardiovascular disease and/or vascular risk factors after the end of quarantine and compare it with the prevalence during quarantine and before the COVID-I9 pandemic. Methods: An online questionnaire was sent I50 days after the quarantine ended. The Hospital Anxiety Depression (HAD) scale was used and clinical and demographic data were recorded. The results were compared with samples obtained during quarantine and the EPICA study conducted in 2016 before the COVID-19 pandemic. Results: The samples included 1076, 3542 and 1035 patients respectively. The prevalence of anxiety fluctuated but did not change (20.8\%, $13.5 \%$ and $21 \%$ respectively). Depression increased its prevalence $(9.8 \%, 16.7 \%$ and $19.7 \%$ respectively). Depression was associated with coronary heart disease and risk behaviors (cigarette smoking and sedentary lifestyle). Conclusion: Depression doubled its prevalence during the COVID-I 9 pandemic in patients with cardiovascular disease and/or vascular risk factors. The effects of the pandemic appear to extend beyond the duration of the quarantine.
\end{abstract}

Keywords: Anxiety - Depression - COVID-19 - HAD - Cardiovascular disease.

RECIBIDO 7/7/2021 - ACEPTADO |3/9/2021

1. Unidad Corazón y Cerebro. Departamento de Epidemiología y Prevención Cardiovascular. Instituto Cardiovascular de Buenos Aires (ICBA), Argentina.

Autor de referencia:

Augusto Vicario

avicario@icba.com.ar

augusto.vicario@gmail.com

Lugar de realización del estudio: Instituto Cardiovascular de Buenos Aires (ICBA), Argentina. 


\section{Introducción}

Las emociones negativas, tales como el miedo, la tristeza, la desesperanza o la angustia presentes durante la cuarentena o "Aislamiento Social Preventivo y Obligatorio" (ASPO, como fue denominado en Argentina) implementado por la pandemia del Coronavirus Disease 2019 (COVID-19), impactaron sobre la salud mental de las personas más vulnerables. Diversas publicaciones dieron cuenta del aumento en la prevalencia de trastornos psicológicos y/o neuropsiquiátricos como consecuencia de la estricta cuarentena (Qiu et al., 2020; Brooks et al., 2020) o de la infección por COVID-19 (Taquet et al., 2021). Una encuesta realizada por nuestro grupo de investigación en una muestra de personas con enfermedad cardiovascular y/o factores de riesgo vasculares en el día 90 del ASPO reveló que el $13.5 \%$ presentaba ansiedad y el $16.7 \%$ depresión (Vicario et al., 2020). Estos porcentajes contrastan con los obtenidos en el estudio realizado en población general en la Argentina que arrojaron 16,4\% y 12,3\% respectivamente, medidos con la Entrevista Internacional Compuesta (CIDI)) (Stagnaro et al., 2018) y en poblaciones con enfermedad cardiovascular $(20.8 \%$ y $9.8 \%$ respectivamente) (Cerezo et al., 2018).

El desconocimiento sobre la pandemia COVID-19 hace que ignoremos cómo impacta la enfermedad sobre las funciones afectivas y cuáles podrían ser las consecuencias a largo plazo sobre la salud mental no solo de aquellos que se contagiaron el SARS-CoV-2, desarrollaron la enfermedad y se recuperaron, sino también de quienes solo soportaron las políticas sanitarias de restricciones impuestas por la pandemia (aislamiento social). Es así que la pandemia COVID-19 debiera ser considerada como un "evento traumático" para la salud mental, homologable a las catástrofes naturales, los conflictos bélicos, los ataques terroristas u otras epidemias pasadas, responsable de la persistencia de síntomas psicológicos o del aumento en la prevalencia de trastornos conductuales como la ansiedad y/o la depresión. Por lo cual, el objetivo de la presente investigación, realizada 150 días después de finalizar el ASPO, fue explorar las consecuencias a largo plazo sobre la salud mental (prevalencia de ansiedad y depresión) y compararla con dos muestras previas (antes de la pandemia COVID-19 y durante el ASPO) realizadas con la misma metodología a fin de conocer la trayectoria de los trastornos neuropsiquiátricos en el tiempo y el impacto que pudo causar tanto el ASPO prolongado implementado en nuestro país (7 meses y 6 días) como la enfermedad COVID-19.

\section{Métodos}

En la presente muestra fueron analizados los datos de 1076 cuestionarios (encuestas) completos. La metodología utilizada fue descripta en extenso en dos publicaciones previas (Vicario et al., 2020; Cerezo et al., 2018). En forma resumida, se utilizó la escala de Ansiedad-Depresión Hospitalaria (HAD, por sus siglas en inglés), considerando como puntos de corte: 0-7 puntos "normal", 8-10 puntos: "caso probable" y 11-21 puntos: "caso" de ansiedad y/o depresión según el artículo original de Zigmond y Snaith (1983). El cuestionario (diseñado sobre una plataforma Jot Form) registró los datos clínicos (hipertensión, diabetes, dislipidemia, enfermedad cardiovascular, etc.) y demográficos (edad, sexo). A diferencia de las dos encuestas anteriores se agregaron 2 preguntas referidas a la enfermedad COVID-19 (contagio, internación, requerimiento de oxígeno, etc.). El cuestionario fue enviado el 07 de abril de 2021 y re-enviado en forma automática el 09 de abril de 2021 (a los 150 días de finalizado el ASPO) a las direcciones de correo electrónico de la base de datos del Instituto Cardiovascular de Buenos Aires (ICBA) (personas $\geq 16$ años de edad) que respondieron la encuesta anterior realizada durante el ASPO $(n=4.316)$ (Vicario et al., 2020). Se obtuvo una tasa de respuesta del $41.5 \%$ $(\mathrm{n}=1320)$ y una tasa de retención del $82.2 \%(\mathrm{n}=1086)$. Fueron excluidas 10 respuestas por no haber aceptado el consentimiento informado.

La encuesta anterior se realizó 3 meses después que las autoridades de Argentina decretaran el ASPO por la pandemia COVID-19 (día 90 del ASPO, 20 de junio de 2020) (Vicario et al., 2020). Esta fue enviada a 45.088 direcciones de correo electrónico pertenecientes a pacientes con enfermedades cardiovasculares y/o factores de riesgo vascular, $\geq 16$ años de edad, atendidos en el ICBA, entre 2018 y 2019. La tasa de respuesta fue $21.5 \%$ y la tasa de retención $14.1 \%(n=3542)$. Para comparación de las dos encuestas realizadas durante la pandemia COVID-19 se utilizaron los resultados del estudio EPICA (Estudio sobre la prevalencia de depresión en pacientes cardiovasculares) (Cerezo et al., 2018), realizado entre octubre y noviembre de 2016. En aquella oportunidad fueron encuestados, en forma voluntaria, en la sala de espera de los consultorios de cardiología del ICBA, pacientes $\geq 16$ años edad con enfermedad cardiovascular y/o factores de riesgo vascular $(n=1035)$. En las tres muestras, las encuestas fueron no personalizadas, no hubo incentivos, fueron auto-administradas y se utilizó la misma escala para medir la ansiedad y la depresión (HAD), y el mismo cuestionario para registrar los datos clínicos y demo- 
gráficos. La escala HAD está validada para el idioma español y presenta rangos de sensibilidad entre 0,74 y 0,84 y de especificidad entre 0,78 a 0,80 . Además, es una escala que sustituye los síntomas somáticos que pueden presentar los pacientes con enfermedades cardiovasculares por otros específicos de la esfera psíquica. Así quedaron conformadas tres muestras de pacientes $\geq 16$ años edad, con enfermedad cardiovascular y/o factores de riesgo vascular atendidos en la misma institución cardiológica: primera encuesta "antes de la pandemia COVID-19" ( $\mathrm{n}=1035)$, segunda encuesta "durante el ASPO" $(\mathrm{n}=3542)$ y tercera encuesta "después del ASPO" (n-1076).

El diseño del estudio fue de corte transversal y descriptivo. Las variables categóricas se expresaron en porcentajes y las continuas por su media \pm desvío estándar (DE). Para las comparaciones se utilizó el test de chi2 ajustado o Mann Whitney de acuerdo a la naturaleza de las variables. En todos los casos se trabajó con un nivel de confianza del 95\%. El estudio cumplió con todas las reglamentaciones locales e internacionales vigentes y respetó las normas éticas para los estudios de investigación en seres humanos. El protocolo del estudio así como el consentimiento informado fue aprobado por el Comité de Ética Independiente del ICBA (código de registro: 4270). Todos los encuestados firmaron el consentimiento informado para participar del estudio y las respuestas fueron procesadas en forma anónima no siendo posible asociar los datos con las personas que los originaron cumpliendo con la ley nacional de protección de datos personales (Ley No 25.326).

\section{Resultados}

Un total de 1076 participantes completaron la encuesta. La tabla 1 resume las características clínicas y demográficas de la muestra estudiada. La edad promedio de la muestra fue 63.3 \pm 12.1 años (rango 21-95 años) $y$ el $59.8 \%(n=644)$ fueron mujeres. Se registraron 91 casos de COVID-19 (8.45\%), el 64.8\% fueron mujeres $(\mathrm{n}=59)$ y el $12 \%$ fueron formas moderadas a graves que requirieron internación y suplemento de oxígeno (ningún caso requirió internación en la unidad de cuidados intensivos ni asistencia ventilatoria mecánica).

La prevalencia en la muestra total de "caso" de ansiedad fue $21 \%(\mathrm{n}=227)$ y "caso" de depresión $19.7 \%$, siendo más frecuente en las mujeres (ansiedad 25.6\% vs. $14.3 \%, \mathrm{p}<0.0001$; depresión $22.0 \%$ vs. $16.2 \%$, p 0.019 ). No hubo diferencias en la prevalencia de ansiedad y depresión entre contagiados (COVID +) y no contagiados (COVID -) (ansiedad $19.7 \%$ vs $21 \%$, p 0.767 y depresión $19.7 \%$ vs $19.7 \%$, p 0.986). No hubo diferencia estadística entre los puntajes de la escala de depresión entre categorías de edad, en tanto la ansiedad fue más prevalente en jóvenes (categoría $\leq 40$ años). El mayor puntaje en la escala de ansiedad (9.3 \pm 5.4 puntos) fue en la categoría $\leq 40$ años y el menor $(5.2 \pm 4.3$ puntos) en la categoría $\geq 71$ años (ver tabla 2).

La tabla 3 muestra las diferencias absolutas y relativas en la prevalencia de ansiedad y depresión en las tres muestras examinadas. Las características clínicas y demográficas de la muestra pre-pandemia y la muestra durante el ASPO fueron descriptas en sus respectivas publicaciones (Cerezo et al., 2018; Vicario et al., 2020). La prevalencia de depresión se duplicó (diferencia relativa 2.0) durante la pandemia COVID-19 comparada con la prevalencia pre-pandemia. En tanto la prevalencia de ansiedad presentó un comportamiento fluctuante, descendió durante el ASPO (cuarentena) para luego retornar a la prevalencia pre-pandemia (20.0\%, $13.5 \%$ y $21 \%$ respectivamente).

La tabla 4 muestra las características demográficas de las tres muestras y la asociación con la depresión en cada una de ellas. La edad, el riesgo de fumar y la inactividad física se asociaron con los "casos" de depresión en las tres muestras. El sedentarismo presentó una relación dosis-respuesta con la severidad del síntoma depresión: antes de la pandemia COVID-19 (sin depresión $42.8 \%$, probable $56.1 \%$ y depresión $71.5 \%$ ), durante el ASPO (sin depresión 61.2\%, probable $81.1 \%$ y depresión $86.1 \%$ ) y pos ASPO (sin depresión $55.1 \%$, probable $66.1 \%$ y depresión $77.3 \%$ ). En tanto la depresión se asoció con la enfermedad coronaria en la segunda muestra realizada durante el ASPO (enfermedad coronaria p 0.03 , infarto de miocardio $<0.0001 \mathrm{y}$ angioplastia 0.05) y en la primera muestra pre-pandemia, pero solo en el sexo masculino. El 71.5\% de la muestra presentó algún trastorno del sueño, el 31.1\% consumía psicofármacos y el $12.8 \%$ incrementó la dosis durante la pandemia. Los participantes con ansiedad "caso" y depresión "caso" realizaron más consultas médicas en los servicios de emergencia o en los consultorios médicos (ansiedad $45.8 \%$ vs $18.8 \%$ y depresión $40.5 \%$ vs $20.6 \%$ respectivamente, $\mathrm{p}<0.0001$ ).

\section{Discusión}

El hallazgo más relevante del presente estudio fue la elevada prevalencia de ansiedad y depresión observada 150 días después de haber concluido el período de ASPO (21\% y $19.7 \%$ respectivamente). En esta muestra, el elevado porcentaje de ansiedad y depresión podría atribuirse a los efectos traumáticos de la pandemia COVID-19 (pérdida de familiares y amigos por la 
Tabla I. Características clínicas y demográficas de la muestra pos ASPO (n=1076)

\begin{tabular}{|c|c|c|c|c|}
\hline Variable & Totales & Femenino & Masculino & Valor $p$ \\
\hline Muestra & 1076 & 644 & 431 & \\
\hline Edad (años-media $\pm D E$ ) & $63.3 \pm 12.1$ & $61.8 \pm 12.4$ & $65.7 \pm 11.2$ & 0.000 \\
\hline Rango (edad) & $21-95$ & $21-95$ & $26-90$ & \\
\hline Género femenino (\%) & & $59.8 \%$ & $40.2 \%$ & 0.000 \\
\hline \multicolumn{5}{|l|}{ Factores de riesgo vascular } \\
\hline Hipertensión & $44.7(482)$ & $38.1(246)$ & $54.7(236)$ & 0.000 \\
\hline Diabetes & $11.6(125)$ & $6.9(45)$ & $18.5(80)$ & 0.000 \\
\hline Dislipidemia & $16.0(173)$ & $16.7(108)$ & $15.0(65)$ & 0.460 \\
\hline Fumador & $6.3(68)$ & $7.1(46)$ & $4.8(2 \mathrm{I})$ & 0.131 \\
\hline Sedentario & $61.5(662)$ & $64.9(418)$ & $56.6(244)$ & 0.006 \\
\hline \multicolumn{5}{|c|}{ Enfermedades cardiovasculares } \\
\hline Enfermedad Coronaria (*) & $19.5(210)$ & $7.2(47)$ & $37.8(163)$ & 0.000 \\
\hline IM & $6.9(75)$ & $2.7(18)$ & $13.2(57)$ & 0.000 \\
\hline CRM & $9.0(97)$ & $2.4(16)$ & $18.7(81)$ & 0.000 \\
\hline ATC (con o sin stent) & $10.6(115)$ & $3.5(23)$ & $21.3(92)$ & 0.000 \\
\hline FA & $5.0(54)$ & $3.4(22)$ & $7.4(32)$ & 0.003 \\
\hline $\mathrm{ACV}$ & $2.3(25)$ & $1.7(1 \mathrm{I})$ & $3.2(14)$ & 0.101 \\
\hline \multicolumn{5}{|c|}{ Característica enfermedad COVID-I9 } \\
\hline Contagios COVID-19 & $8.4(91)$ & $61.5(59)$ & $35.1(32)$ & 0.000 \\
\hline Aislamiento domiciliario & $87.9(80)$ & $62.5(50)$ & $37.5(30)$ & 0.002 \\
\hline Requerimiento $\mathrm{O}_{2}$ & $12.0(11)$ & $81.8(9)$ & 18.1 (2) & 0.003 \\
\hline \multicolumn{5}{|l|}{ Trastornos afectivos } \\
\hline Ansiedad (probable) & $15.3(165)$ & $18.0(116)$ & II.3 (49) & 0.003 \\
\hline Ansiedad "caso" & $21.0(227)$ & $25.6(165)$ & $14.3(62)$ & 0.000 \\
\hline Depresión (probable) & $18.1(195)$ & $21.5(139)$ & $12.9(56)$ & 0.000 \\
\hline Depresión “caso” & $19.7(2 \mid 2)$ & $22.0(142)$ & $16.2(70)$ & 0.019 \\
\hline
\end{tabular}

Abreviaturas: (*) representa la suma de pacientes con infarto de miocardio, angioplastia y cirugía de revascularización; IM: infarto de miocardio; CRM: cirugía de revascularización miocárdica;ATC: angioplastia transluminal coronaria; FA: fibrilación auricular;ACV: ataque cerebrovascular.

Tabla 2. Puntajes de ansiedad y depresión (escala HAD) por categoría de edad

\begin{tabular}{lcccccc}
\hline Categoría por edad & $\leq \mathbf{4 0}$ & $\mathbf{4 I - 5 0}$ & $\mathbf{5 I - 6 0}$ & $\mathbf{6 I - 7 0}$ & $\mathbf{2 7}$ & Valor $\mathbf{p}$ \\
\hline $\mathrm{n}$ & 52 & $1 \mathrm{I}$ & 228 & 358 & 324 & \\
\hline HAD-Ansiedad & $9.3 \pm 4.5$ & $7.0 \pm 4.8$ & $7.7 \pm 4.4$ & $5.8 \pm 4.5$ & $5.2 \pm 4.3$ & 0.002 \\
\hline HAD-Depresión & $6.6 \pm 4.4$ & $6.6 \pm 4.8$ & $6.8 \pm 4.5$ & $6.3 \pm 4.8$ & $6.2 \pm 4.3$ & $\mathrm{~ns}$ \\
\hline
\end{tabular}

Puntaje de ansiedad: la categoría $\leq 40$ años es el mayor puntaje diferente a todos. La categoría $\geq 7 \mathrm{I}$ años es el menor puntaje diferente a todos. La categoría 4I-50 años y 5I-60 años son iguales y diferentes al resto. La categoría 6 I-70 años y $\geq 7 \mathrm{I}$ años son iguales y diferentes al resto. Puntaje de depresión: No hubo diferencias con significación estadística en los puntajes de depresión entre categorías de edad. 
Tabla 3. Características demográficas y prevalencia de ansiedad y depresión en las 3 muestras antes de la pandemia COVID-19 y durante y después del ASPO

\begin{tabular}{|c|c|c|c|c|c|c|}
\hline \multirow{2}{*}{\multicolumn{2}{|c|}{ Variable }} & \multirow{2}{*}{$\begin{array}{l}\text { Antes } \\
\text { COVID- I } 9^{[a]}\end{array}$} & \multirow{2}{*}{$\begin{array}{c}\text { Durante } \\
\text { ASPO COVID- } 19^{[b]}\end{array}$} & \multirow{2}{*}{$\begin{array}{c}\text { Después } \\
\text { ASPO COVID-19[c] }\end{array}$} & \multicolumn{2}{|c|}{ Diferencia $^{[a-c]}$} \\
\hline & & & & & Absoluta & Relativa \\
\hline Muestra & & 1035 & 3542 & 1076 & & \\
\hline \multirow[t]{3}{*}{ Género (\%) } & & $44.6 \%$ & $54.7 \%$ & $59.8 \%$ & & \\
\hline & Hombres & $573(55.3)$ & $1602(45.2)$ & $432(40.2)$ & & \\
\hline & Mujeres & $462(44.6)$ & $1940(54.7)$ & $644(59.8)$ & & \\
\hline Edad (años-media \pm DE) & & $61.2 \pm 0.4$ & $60.2 \pm 14$ & $63.3 \pm 12$ & & \\
\hline \multicolumn{7}{|l|}{ Trastornos afectivos } \\
\hline Sin ansiedad & & $611(59.0)$ & $2482(70.0)$ & $684(63.5)$ & 4.5 & 1.0 \\
\hline Ansiedad (probable) & & $208(20.0)$ & $580(16.3)$ & $165(15.3)$ & -4.7 & -0.7 \\
\hline Ansiedad “caso” & & $216(20.8)$ & $480(13.5)$ & $227(21.0)$ & 0.2 & 1.0 \\
\hline Sin depresión & & $794(76.7)$ & $2314(65.3)$ & $699(62.1)$ & -14.7 & -0.8 \\
\hline Depresión (probable) & & $139(13.4)$ & $636(17.9)$ & $195(18.1)$ & 4.7 & 1.3 \\
\hline Depresión “caso" & & $102(9.8)$ & $592(16.7)$ & $212(19.7)$ & 9.9 & 2.0 \\
\hline
\end{tabular}

Tabla 4. Características demográficas de las tres muestras (antes de la pandemia COVID-19 (estudio EPICA), durante y después del ASPO. Comparación de la muestra total con los "casos" de depresión

\begin{tabular}{|c|c|c|c|c|c|c|c|c|c|}
\hline \multirow{2}{*}{$\begin{array}{l}\text { Variable } \\
\text { Muestra }\end{array}$} & \multicolumn{3}{|c|}{$\begin{array}{c}\text { Antes COVID-19 (EPICA) } \\
\text { Nro. (\%) }\end{array}$} & \multicolumn{3}{|c|}{$\begin{array}{c}\text { Durante ASPO-COVID-19 } \\
\text { Nro. (\%) }\end{array}$} & \multicolumn{3}{|c|}{$\begin{array}{c}\text { Después ASPO-COVID-19 } \\
\text { Nro. (\%) }\end{array}$} \\
\hline & $\begin{array}{c}\text { Total } \\
(n=1035)\end{array}$ & $\begin{array}{l}\text { Depresión } \\
\text { "Caso" } \\
(n=102)\end{array}$ & Valor $\mathrm{p}$ & $\begin{array}{c}\text { Total } \\
(n=3542)\end{array}$ & $\begin{array}{l}\text { Depresión } \\
\text { “Caso” } \\
\text { (n=592) }\end{array}$ & Valor $\mathrm{p}$ & $\begin{array}{c}\text { Total } \\
(n=1076)\end{array}$ & $\begin{array}{l}\text { Depresión } \\
\text { “Caso" } \\
(n=2 \mid 2)\end{array}$ & Valor $\mathrm{p}$ \\
\hline $\begin{array}{l}\text { Edad } \\
\text { (años-media } \pm D E \text { ) }\end{array}$ & $61.2 \pm 0.4$ & $63.1 \pm 13.2$ & $<0.0001$ & $60.2 \pm 14$ & $65.7 \pm 11.2$ & $<0.0001$ & $63.3 \pm 12.1$ & $62.7 \pm 12.1$ & 0.50 \\
\hline Rango (edad) & $18-92$ & $32-83$ & & $18-99$ & $26-90$ & & $21-95$ & $22-95$ & \\
\hline $\begin{array}{l}\text { Género femenino } \\
(\%-n)\end{array}$ & $462(44.6)$ & $47(46)$ & 0.78 & $1940(54.7)$ & $372(40.2)$ & $<0.0001$ & $644(59.8)$ & $142(66.9)$ & 0.05 \\
\hline \multicolumn{10}{|c|}{ Factores de riesgo vascular } \\
\hline Hipertensión & $56 \mathrm{I}(54.2)$ & $65(63.7)$ & 0.06 & $1362(38.4)$ & $212(35.8)$ & 0.22 & $482(44.7)$ & $108(50.9)$ & 0.10 \\
\hline Diabetes & $158(15.2)$ & $16(15.6)$ & 0.91 & $355(10.0)$ & $61(10.3)$ & 0.99 & I25 (I I.6) & $26(12.2)$ & 0.78 \\
\hline Dislipidemia & $337(32.7)$ & $32(31.3)$ & 0.80 & $518(14.6)$ & $104(17.5)$ & 0.06 & $173(16.0)$ & $49(23.1)$ & 0.01 \\
\hline Fumador & $103(9.9)$ & $20(19.6)$ & 0.003 & $25 I(7.0)$ & $58(9.7)$ & 0.02 & $68(6.3)$ & $21(9.9)$ & 0.06 \\
\hline Sedentario & $473(45.7)$ & $73(7 \mid .5)$ & $<0.0001$ & $2497(70.4)$ & $510(86.1)$ & $<0.0001$ & $662(61.5)$ & $164(77.3)$ & $<0.0001$ \\
\hline \multicolumn{10}{|c|}{ Enfermedades cardiovasculares } \\
\hline $\begin{array}{l}\text { Enfermedad } \\
\text { Coronaria }(*)\end{array}$ & $375(36.3)$ & $42(4 I .1)$ & 0.32 & $605(17.0)$ & $80(13.5)$ & 0.03 & $210(19.5)$ & $37(17.4)$ & 0.48 \\
\hline IM & $332(32.0)$ & $39(38.2)$ & 0.20 & $184(5.1)$ & $35(5.9)$ & $<0.0001$ & $75(6.9)$ & $16(7.5)$ & 0.76 \\
\hline CRM & $117(11.3)$ & $17(16.6)$ & 0.10 & $266(7.5)$ & $33(5.5)$ & 0.09 & $97(9.0)$ & $18(8.4)$ & 0.80 \\
\hline ATC/ stent & $185(17.8)$ & $20(19.6)$ & 0.66 & $351(9.9)$ & $44(7.4)$ & 0.05 & $115(10.6)$ & $20(9.4)$ & 0.58 \\
\hline FA & & & & $157(4.4)$ & $28(4.7)$ & 0.74 & $54(5.0)$ & $12(5.6)$ & $12(5.6)$ \\
\hline $\mathrm{ACV}$ & & & & $54(1.5)$ & $9(1.5)$ & 0.99 & $25(2.3)$ & $8(3.7)$ & 0.22 \\
\hline
\end{tabular}

Abreviaturas: (*) Representa la suma de infarto de miocardio, angioplastia y cirugía de revascularización. IM: infarto de miocardio; CRM: cirugía de revascularización miocárdica;ATC: angioplastia transluminal coronaria; FA: fibrilación auricular;ACV: ataque cerebrovascular. 
enfermedad COVID-19) y sus consecuencias sociales y económicas (restricciones a la circulación, aislamiento social, caída de la economía, pérdida laboral, etc.) más que a la enfermedad COVID-19. Habida cuenta que, según nuestros resultados, la prevalencia de ansiedad y depresión no fue más elevada en personas que se contagiaron con el SARS-CoV-2 y desarrollaron la enfermedad (si bien en la muestra analizada no hubo formas graves de la enfermedad o que requirieran internación en unidades de cuidados intensivos). En línea con estos resultados, otro estudio informó una prevalencia de ansiedad 6 meses después de la infección por COVID-19 entre el 17\%-39\%, no limitado a las formas graves (Taquet et al., 2021). Coincidente con otras investigaciones (Qiu et al., 2020; Rogers et al., 2020) y nuestras encuestas previas, la prevalencia de ansiedad y depresión continúa siendo más elevada en el género femenino. En cuanto a las categorías por edad, la ansiedad fue más prevalente en los jóvenes (categoría de $\leq 40$ años), en tanto la prevalencia de depresión no mostró diferencias entre las categorías de edad en la muestra pos ASPO. Comparada con la muestra realizada durante el ASPO, la ansiedad mostró el mismo comportamiento, siendo más prevalente en los jóvenes (categoría $\leq 40$ años), pero la depresión fue más prevalente en los adultos mayores ( $\geq 71$ años). Si bien son tres muestras distintas (no se siguió a la misma población), todas las personas incluidas presentaban enfermedad cardiovascular y/o factores de riesgo y todos fueron atendidos e integran la base de datos del ICBA. La comparación de la presente encuesta con las dos encuestas previas (Figura 1) muestra un aumento sostenido en la prevalencia de depresión y un comportamiento fluctuante en la prevalencia de ansiedad.

Figura I. Prevalencia de ansiedad y depresión en las 3 muestras, antes de la pandemia COVID-19, durante y después del ASPO (cuarentena)

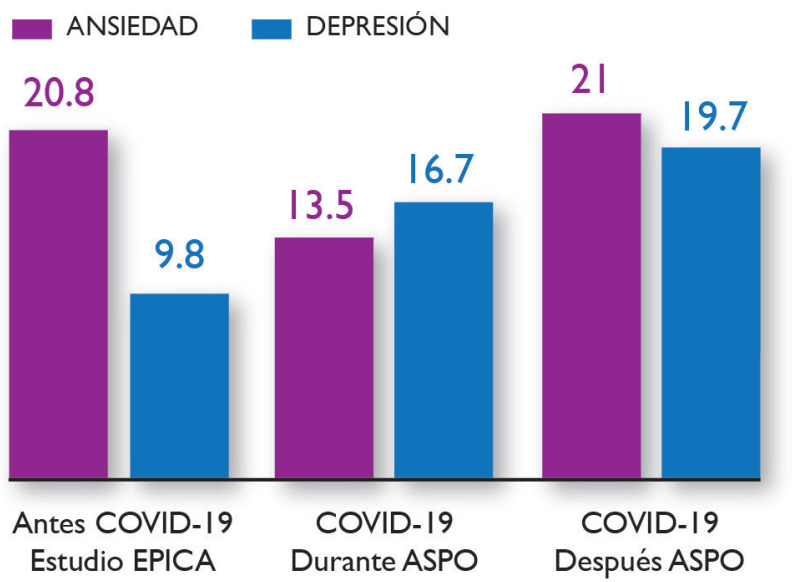

Un resultado similar se observó cuando se categorizó a los participantes de acuerdo a la intensidad del síntoma ansiedad y depresión (puntaje de la escala HAD) (Figura 2).

Figura 2. Prevalencia de ansiedad y depresión de acuerdo al puntaje obtenido en la escala HAD (Antes de la pandemia COVID-19, durante y después del ASPO)
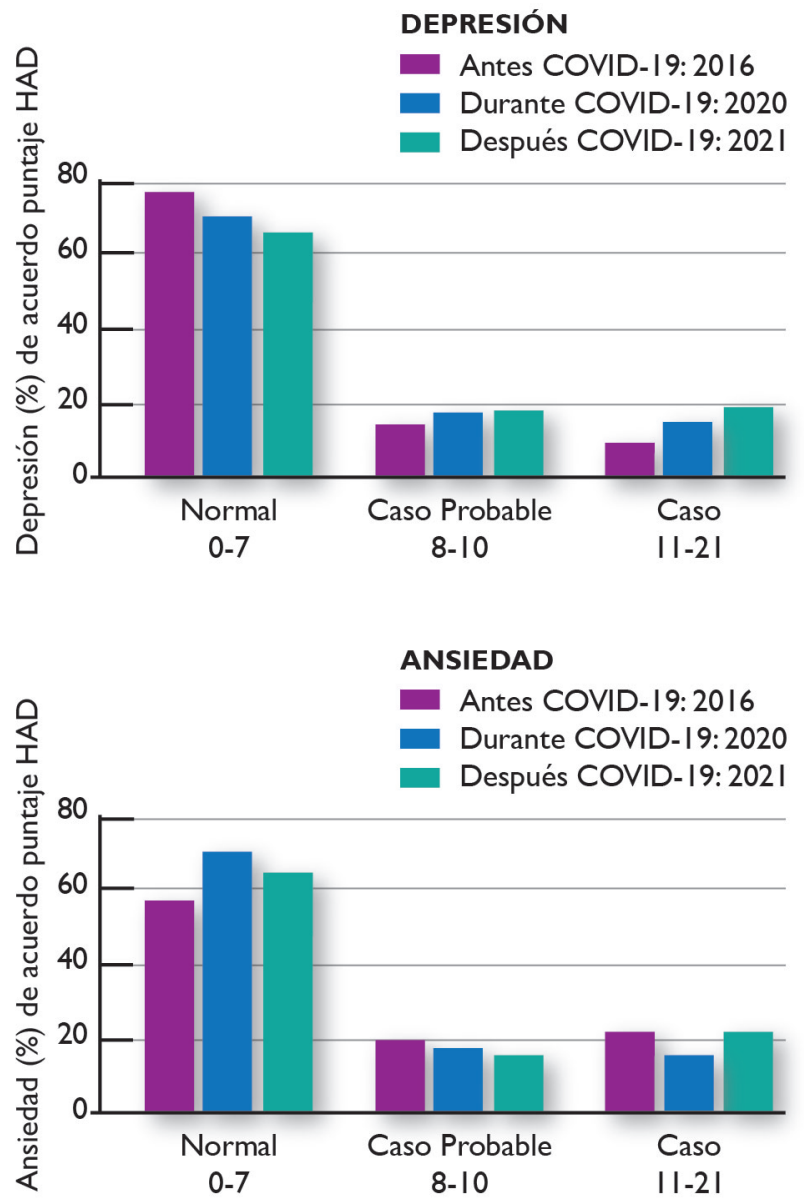

Estas diferencias podrían ser explicadas por la "carga" de emociones negativas (incertidumbre, miedo) generadoras de ansiedad que fueron variando en el tiempo de acuerdo a las características cambiantes de la pandemia (número de contagios y fallecimientos, estatus sanitario, "olas de contagio", disponibilidad de vacunas, etc.).

La ansiedad fue elevada en pacientes con enfermedad cardiovascular antes de la pandemia COVID-19 (20.8\%), disminuyó a los 3 meses del confinamiento $(13,5 \%)$, período en el cual en Argentina el impacto de la pandemia (contagios y fallecimientos) era muy bajo y aumentó en la última encuesta (19.7\%), período coincidente con el inicio de la "segunda ola de contagios" y una profunda crisis económica. En tanto los síntomas 
depresivos (desesperanza, anhedonia y tristeza) han presentado un aumento sostenido relacionado, tal vez, a variables que se mantuvieron constantes durante la pandemia, tales como el aislamiento social, la movilidad restringida, el fallecimiento de familiares, la crisis económica y social y la pérdida laboral, entre otros.

Desde hace más de dos décadas se ha reconocido a la depresión como un factor de riesgo independiente para la enfermedad coronaria, con efecto dosis-respuesta y un indicador pronóstico de mortalidad, con un riesgo atribuido a la enfermedad, similar a la hipertensión arterial (Ford et al., 1998). A su vez, la depresión se asocia con conductas de riesgo (inactividad física, consumo de cigarrillos, obesidad y mala adherencia a tratamientos) que predisponen, en forma indirecta, a la enfermedad cardiovascular. Es así que, la American Heart Association en forma conjunta con la American Psychiatric Association ha recomendado su tamizaje en la evaluación del riesgo cardiovascular de los pacientes (Lichtman et al., 2008; Lichtman, et al., 2014). Nuestras observaciones, coincidentes con otros estudios (Ettman et al., 2020), muestran que la prevalencia de depresión se duplicó respecto a la prevalencia estimada antes de la pandemia COVID-19 (9.8\% a $19.7 \%$, diferencia relativa 2.01 ) y se cuadriplicó con respecto a las estimaciones en la población general $(4.7 \%$ a $19.7 \%$, diferencia relativa 4.19$)$. En las tres muestras estudiadas hubo una asociación significativa con dos factores de riesgo o conductas de riesgo: el fumar cigarrillos y el sedentarismo o actividad física $<150$ minutos semanales. En los tres casos existió una relación dosis-respuesta entre el nivel de sedentarismo y el puntaje de la escala HAD-depresión, siendo las cifras más elevadas las observadas en la muestra obtenida durante el ASPO, explicada, tal vez, por las mayores restricciones a la movilidad implementadas en ese período. En tanto, los otros factores de riesgo (hipertensión, diabetes, dislipemia) no encontraron asociación alguna con el puntaje de la escala HAD (ansiedad y/o depresión). Finalmente, la asociación entre la enfermedad coronaria y la depresión solo fue observada en el sexo masculino en la muestra anterior a la pandemia COVID-19 y en ambos sexos en la muestra obtenida durante el ASPO. La muestra actual (post ASPO) no encontró asociación estadística, pudiendo este comportamiento ser explicado por el tamaño muestral.
Nuestro estudio plantea algunas limitaciones que debemos mencionar. La primera, responde a las características de los datos obtenidos a través de encuestas on-line. Sin embargo, en las tres encuestas la tasa de retención (respuestas completas) alcanzada ha sido muy elevada. La segunda limitación podría atribuirse a la comparación realizada entre tres estudios de corte transversal (antes de la pandemia COVID-19, durante y después del ASPO) al encuestar individuos diferentes (no se hizo un seguimiento de los mismo individuos en el tiempo). No obstante, se utilizó la misma herramienta validada (escala HAD) para tamizar los síntomas de ansiedad y depresión (si bien se sabe que el diagnóstico de depresión y/o ansiedad debe ser clínico), se encuestaron personas $\geq 16$ años de edad con enfermedad cardiovascular y/o factores de riesgo vascular asistidas en la misma institución (ICBA) y en todos los casos el cuestionario fue voluntario, auto-administrado y sin incentivos.

\section{Conclusión}

En suma, la pandemia COVID-19 y sus consecuencias físicas, sociales y económicas ha impactado en forma negativa sobre la salud mental de las personas. La prevalencia de los trastornos depresivos aumentó en forma considerable siendo, en muchas circunstancias, asociada con síntomas de ansiedad. Aún desconocemos si son solo trastornos o síntomas reactivos y temporarios a la pandemia, a las medidas sanitarias implementadas o constituyen un cambio en la prevalencia de la depresión. No obstante, cualquiera sea la explicación, este crecimiento acelerado plantea un desafío para la medicina clínica que deberá abordar la problemática en forma multidisciplinaria, entendiendo que la salud mental y las enfermedades cardiovasculares mantienen una relación bidireccional.

Conflictos de intereses: los autores declaran no tener conflictos de intereses ni recibieron financiación para realizar el presente trabajo.

Agradecimientos: Los autores quieren expresar su agradecimiento a la Dra. Ruth Henquin, presidente del Comité de Ética e Investigación del Instituto Cardiovascular de Buenos Aires (ICBA) y al Sr. Nicolás Berney, miembro del Departamento de Comunicaciones del Instituto Cardiovascular de Buenos Aires (ICBA). 


\section{Referencias bibliográficas}

Brooks, S.K., Webster, R.K., Smith, L.E., Woodland, L., Wessely, S., Greenberg, N., et al. (2020). The psychological impact of quarantine and how to reduce it: rapid review of the evidence. Lancet, 395(10227): 912-920. doi: https://doi.org/10.1016/S0140-6736(20)30460-8

Cerezo, G.H., Vicario, A., Fernández, R., Enders, J. (2018). Prevalencia de depresión y ansiedad en la consulta cardiológica. Estudio EPICA. (Estudio sobre la prevalencia de depresión en pacientes cardiovasculares). Rev Fed Arg Cardiol, 47(1):26-31.

Ettman, C.K., Abdalla, S.M., Cohen, G.H., Sampson, L., Vivier, P.M., Galea, S. (2020). Prevalence of Depression Symptoms in US Adults Before and During the COVID-19 Pandemic. JAMA Netw Open; 3(9):e2019686. doi:10.1001/jamanetworkopen.2020.196

Ford, D.E., Mead, L.A., Chang, P.P., Cooper-Patrick, L., Wang, N.Y., Klag, M.J. (1998). Depression is a risk factor for coronary artery disease in men: the precursors study. Arch Intern Med, 158(13):1422-6.

Lichtman, J.H., Bigger, J.T. Jr., Blumenthal, J.A., Frasure-Smith, N., Kaufmann, P.G., Lespérance, F., et al.; AHA Prevention Committee of the Council on Cardiovascular Nursing; AHA Council on Clinical Cardiology; AHA Council on Epidemiology and Prevention; AHA Interdisciplinary Council on Quality of Care and Outcomes Research; APA. (2008). Depression and coronary heart disease: recommendations for screening, referral, and treatment: a science advisory from the AHA Prevention Committee of the Council on Cardiovascular Nursing, Council on Clinical Cardiology, Council on Epidemiology and Prevention, and Interdisciplinary Council on Quality of Care and Outcomes Research: endorsed by the APA. Circulation, 118(17):1768-75. doi: 10.1161/CIRCULATIONAHA.108.190769

Lichtman, J.H., Froelicher, E.S., Blumenthal, J.A., Carney, R.M., Doering, L.V., Frasure-Smith, N., Kenneth, E., Freedland, Jaffe, A. S., Leifheit-Limson, E. C., Sheps, D. S., Vaccarino, V., Wulsin, L., and American Heart Association Statistics Committee of the Council on Epidemiology and Prevention and the Council on Cardiovascular and Stroke Nursing. (2014). Depression as a risk factor for poor prognosis among patients with acute coronary syndrome: systematic review and recommendations: a scientific statement from the American Heart Association. Circulation, 129(12):1350-69. https://doi.org/10.1161/CIR.0000000000000019
Qiu, J., Shen, B., Zhao, M., Wang, Z., Xie, B., Xu, Y. (2020). A nationwide survey of psychological distress among Chinese people in the COVID-19 epidemic: implications and policy recommendations. Gen Psychiatr, 33(2): e100213. doi: 10.1136/gpsych-2020-100213

Rogers, J. P., Chesney, E., Oliver, D., Pollak, T.A., McGuire, P., Fusar-Poli, P., et al. (2020). Psychiatric and neuropsychiatric presentations associated with severe coronavirus infections: a systematic review and meta-analysis with comparison to the COVID-19 pandemic. Lancet Psychiatry, 7(7):611627. doi: https://doi.org/10.1016/S2215-0366(20)30203-0

Stagnaro, J. C., Cía, A., Vázquez, N., Vommaro, H., Nemirovsky, M., Serfaty, E., Sustas, S. E., Medina Mora M. E., Benjet, C., Aguilar Gaxiola, S., Kessler, R. (2018). Estudios epidemiológico de salud mental en población general de la República Argentina. Vertex Rev Arg Psiquiatr, 29(142), 275299. PMID: 30785970

Taquet, M., Geddes, J.R., Husain, M., Luciano, S., Harrison, P.J. (2021). 6-month neurological and psychiatric outcomes in 236379 survivors of COVID-19: a retrospective cohort study using electronic health records. Lancet Psychiatry, 8(5):416-427. doi: https://doi.org/10.1016/S2215-0366(21)00084-5

Vicario, A., Fernández, R., Enders, J., Alves de Lima, A., Cerezo, G.H. (2020). Prevalencia de ansiedad y depresión en pacientes con enfermedades cardiovasculares durante la pandemia COVID-19. Rev Fed Arg Cardiol, 49(3):115.

Zigmond, A.S., Snaith, R.P. (1983). The Hospital Anxiety and Depression Scale. Acta Psychiatr Scand, 67:361-70. 